УДК 539.374

СВОЙСТВА ТЕОРЕТИЧЕСКИХ КРИВЫХ ПОЛЗУЧЕСТИ ПРИ СТУПЕНЧАТОМ НАГРУЖЕНИИ В ЛИНЕЙНОЙ ВЯЗКОУПРУГОСТИ

(C) 2015 г. Хохлов А.В.

НИИ механики Московского государственного университета им. М.В. Ломоносова, Москва, Российская Федерация

andrey-khokhlov@ya.ru

Поступила в редакичию 24.06.2015

Изучены общие математические свойства семейств теоретических кривых ползучести при произвольном ступенчатом нагружении, порождаемых линейным интегральным соотношением вязкоупругости с произвольной (возрастающей выпуклой вверх) функцией ползучести. Исследованы их зависимость от характеристик функции ползучести и параметров типичных программ нагружения, точки минимума, интервалы монотонности, скачки в точках разрыва и асимптотика кривых ползучести, условия затухания памяти, влияние перестановки ступеней нагружения и т.п. В частности, выявлена ключевая роль величины предела производной функции ползучести на бесконечности (ее отличие от нуля влечет накопление остаточной деформации и отсутствие затухания памяти). Свойства теоретических кривых ползучести сопоставлены с типичными свойствами экспериментальных кривых вязкоупругопластичных материалов. Выявлены эффекты, которые (не) способно описывать линейное определяющее соотношение, и набор атрибутивных признаков кривых ползучести, наличие которых надо установить у экспериментальных кривых перед попыткой моделирования поведения конкретных (классов) материалов в рамках линейной теории.

Ключевые слова: функции ползучести и релаксации, ступенчатое нагружение, асимптотика кривых ползучести, затухание памяти, регулярные и сингулярные модели, индикаторы адекватности линейной теории.

Эксперименты на ползучесть при ступенчатом нагружении (с кусочно-постоянным напряжением) - важный вид квазистатических испытаний, позволяющий уловить и обследовать разные аспекты поведения материала, детали реализации многих эффектов, собрать более богатую информацию для идентификации и верификации моделей и выявления лучшей из них по сравнению с кривыми ползучести (КП) при постоянном напряжении (такие КП все модели описывают адекватно при правильной настройке).

Эта статья - продолжение цикла работ по качественному анализу линейного определяющего соотношения (ОС) вязкоупругости. В [1-6] выведены в общем виде уравнения семейств всех основных теоретических кривых интегрального ОС с произвольными функциями релаксации (ФР) и ползучести (ФП) (кривых деформиро- 
вания при постоянных скоростях нагружения и деформации, релаксации и ползучести с произвольной начальной стадией нагружения и др.), детально изучены их общие качественные свойства в зависимости от свойств ФП и ФР. Качественный анализ ОС - важная стадия аттестации любого ОС [7-9], выявления ограничений на материальные функции и списка моделируемых термомеханических эффектов. Цель настоящей статьи - изучение общих свойств семейств теоретических КП при ступенчатом нагружении, порождаемых линейным ОС. Их исследование в общем виде и даже краткий перечень, к сожалению, отсутствуют в монографиях и обзорах по вязкоупругости и механике полимеров, в частности, в [10-20].

В статье приняты следующие сокращения и обозначения: ФР и ФП (КР, КП) функции (кривые) релаксации и ползучести, КОП - кривая обратной ползучести; $\mathrm{h}(t)$ - функция Хевисайда, $\delta(t)$ - дельта-функция Дирака, РеМ - регулярные модели (с ФП: П $(0) \neq 0) ;$ СиМ - сингулярные модели (ФР содержит слагаемое $\eta \delta(t))$; $y(\theta):=y(\theta+)-$ предел функции $y(t)$ справа в точке $t=\theta ; \hat{y}\left(t_{*}\right):=y\left(t_{*}+0\right)-y\left(t_{*}-0\right)-$ величина скачка $y(t)$ в точке $t_{*}$.

\section{1. Некоторые свойства линейного определяющего соотношения вязкоупругости}

Истории напряжения $\sigma(t)$ и деформации $\varepsilon(t)$ связаны в линейной вязкоупругости интегральными операторами, инвариантными относительно сдвигов по времени [10-20]:

$$
\varepsilon(t)=\int_{0}^{t} \Pi(t-\tau) d \sigma(\tau), \quad \sigma(t)=\int_{0}^{t} R(t-\tau) d \varepsilon(\tau), \quad t \geq 0 .
$$

Эти ОС описывают одномерные изотермические процессы в структурно-стабильных материалах. Предполагается, что ФР $R(x)$ и ФП П $(x), x \geq 0,-$ дифференцируемые положительные функции при $x>0$, ФР убывает и выпукла вниз, ФП возрастает и выпукла вверх при $x>0[1,2]$, ФР может иметь особенность в точке $x=0$.

Операторы (1) взаимно обратны, и потому ФП и ФР связаны уравнением

$$
\int_{0}^{t} \Pi(t-\tau) R(\tau) d \tau=t \text { или } \int_{0}^{t} \dot{\Pi}(t-\tau) R(\tau) d \tau+\Pi(0) R(t)=1, \quad t>0 .
$$

Зная ФР, можно найти ФП из уравнения (2) и наоборот $[1,3]$. Поэтому одномерное ОС (1) содержит лишь одну материальную функцию. При П(0) $\neq 0$ (2) и (4) уравнения Вольтерры второго рода с ограниченными (если $\dot{\Pi}(0+)<\infty)$ ядрами, и потому они однозначно разрешимы в пространствах $L_{1}[0, b]$. Случай $\Pi(0)=0$ приводит к уравнению Вольтерры первого рода, некорректным задачам, нерегулярным моделям с особенностью в нуле у ФР, КР и касательного модуля диаграммы деформирования и т.п. [1-6].

Анализ показал, что целесообразно выделять три класса моделей:

$1)$ регулярные - у которых П $(0)>0$ (и тогда $R(0)<\infty)$;

2) сингулярные - с ФР, содержащей слагаемое вида $\eta \delta(t), \eta>0(\Phi P R=\eta \delta(t)$ задает ньютоновскую жидкость с ОС $\sigma=\eta \dot{\varepsilon}$ и входит слагаемым в ФР «половины» реологических моделей - см. ниже);

$3)$ нерегулярные модели с ФР, не содержащей слагаемого $\eta \delta(t)$, но имеющей интегрируемую особенность в точке $t=0(R(0+)=+\infty)$. 
Качественные свойства теоретических кривых моделей трех этих классов заметно отличаются друг от друга [1-6]. Третий класс занимает промежуточное положение между первыми двумя. К нему относится, например, ФР $R(t)=A t^{-\alpha}, \alpha \in$ $\in(0 ; 1)$ (ее ФП имеет вид $\Pi(t)=A^{-1} C(\alpha) t^{\alpha}$ и обладает не только свойством П $(0)=0$, как и СиМ, но и свойством П $(0)=\infty$, переходным к $П(0) \neq 0$, характеризующему $\mathrm{PeM})$.

Операторы (1) определены на линеале кусочно-непрерывных и кусочно-гладких функций. Они представимы в виде: $y(t)=y_{r}(t)+y_{s}(t)$, где $y_{r}(t)-$ регулярная часть (непрерывная функция, $\left.y_{r}(0)=0\right), y_{s}(t)=y(0) \mathrm{h}(t)+\sum_{i=1}^{n} \hat{y}\left(t_{i}\right) \mathrm{h}\left(t-t_{i}\right)-$ ступенчатая функция $\left(i=1, \ldots, n, t_{i+1}>t_{i}\right)$. Тогда

$$
y^{\prime}(t)=y_{r}^{\prime}(t)+y(0) \delta(t)+\sum_{i=1}^{n} \hat{y}\left(t_{i}\right) \delta\left(t-t_{i}\right),
$$

и оператор (1) переводит такой процесс $\sigma(t)$ в деформацию

$$
\varepsilon(t)=\int_{0}^{t} \Pi(t-\tau) \dot{\sigma}_{r}(\tau) d \tau+\sigma(0) \Pi(t)+\sum_{i=1}^{n} \hat{\sigma}\left(t_{i}\right) \Pi\left(t-t_{i}\right) \mathrm{h}\left(t-t_{i}\right), \quad t \geq 0 .
$$

В точках $t_{i}$ отклик $\varepsilon(t)$ имеет скачки $\hat{\varepsilon}\left(t_{i}\right)=\hat{\sigma}\left(t_{i}\right) \Pi(0)$ : так как интегральное слагаемое непрерывно всюду (в силу непрерывности ФП и интегрируемости $\left.\dot{\sigma}_{r}(\tau)\right)$, то $\hat{\varepsilon}\left(t_{i}\right)$ совпадает со скачком слагаемого $\hat{\sigma}\left(t_{i}\right) \Pi\left(t-t_{i}\right) \mathrm{h}\left(t-t_{i}\right)$. Множество точек разрыва $t_{i}$ может быть и бесконечным (как при циклических нагружениях), но не должно иметь предельных точек (тогда в сумму включается только конечное число слагаемых с $\left.t_{i}<t\right)$.

В точках разрыва $\sigma(t)$ разрывна и скорость деформации (даже если $\dot{\sigma}_{r}(t)$ непрерывна):

$$
\begin{aligned}
& \dot{\varepsilon}(t)=\int_{0}^{t} \dot{\Pi}(t-\tau) \dot{\sigma}_{r}(\tau) d \tau+\Pi(0) \dot{\sigma}_{r}(t)+\sigma(0) \dot{\Pi}(t)+ \\
& +\sum_{i=1}^{n} \hat{\sigma}\left(t_{i}\right) \dot{\Pi}\left(t-t_{i}\right) \mathrm{h}\left(t-t_{i}\right)+\sum_{i=1}^{n} \hat{\sigma}\left(t_{i}\right) \Pi\left(t-t_{i}\right) \delta\left(t-t_{i}\right),
\end{aligned}
$$

первое слагаемое всегда непрерывно, третье - если только Пं $(t)$ непрерывна в точке $t_{i}$. Поэтому

$$
\hat{\dot{\varepsilon}}\left(t_{i}\right)=\Pi(0) \hat{\dot{\sigma}}_{r}\left(t_{i}\right)+\sigma(0) \hat{\dot{\Pi}}\left(t_{i}\right)+\sum_{j<i} \hat{\sigma}\left(t_{j}\right) \hat{\dot{\Pi}}\left(t_{i}-t_{j}\right)+\hat{\sigma}\left(t_{i}\right) \dot{\Pi}(0) .
$$

Если Пं( $t)$ непрерывна при $t \geq 0$, то $\hat{\dot{\varepsilon}}\left(t_{i}\right)=\Pi(0) \hat{\dot{\sigma}}_{r}\left(t_{i}\right)+\dot{\Pi}(0) \hat{\sigma}\left(t_{i}\right)$. Если $\hat{\dot{\sigma}}\left(t_{i}\right)=0$ (например, при ступенчатом нагружении), то все же $\hat{\dot{\varepsilon}}\left(t_{i}\right)=\dot{\Pi}(0) \hat{\sigma}\left(t_{i}\right) \neq 0$. Итак, если модель регулярна (то есть $\Pi(0) \neq 0)$ и $\dot{\Pi}(t)$ и $\dot{R}(t)$ непрерывны при $t \geq 0$, то величины скачков в любой точке $t_{*}$ связаны формулами:

$$
\begin{gathered}
\hat{\varepsilon}\left(t_{*}\right)=\hat{\sigma}\left(t_{*}\right) \Pi(0), \quad \hat{\dot{\varepsilon}}\left(t_{*}\right)=\Pi(0) \hat{\dot{\sigma}}\left(t_{*}\right)+\dot{\Pi}(0) \hat{\sigma}\left(t_{*}\right) \text { и } \\
\hat{\sigma}\left(t_{*}\right)=R(0) \hat{\varepsilon}\left(t_{*}\right), \quad \hat{\dot{\sigma}}\left(t_{*}\right)=R(0) \hat{\dot{\varepsilon}}\left(t_{*}\right)+\dot{R}(0) \hat{\varepsilon}\left(t_{*}\right) .
\end{gathered}
$$

Если же модель сингулярна (в ФР присутствует слагаемое $\eta \delta(t))$, то П $(0)=0$ и $\dot{\Pi}(0)=\eta^{-1}[6]$, и потому $\hat{\varepsilon}\left(t_{*}\right)=0, \hat{\dot{\varepsilon}}\left(t_{*}\right)=\hat{\sigma}\left(t_{*}\right) / \eta$, а скачки $\dot{\varepsilon}(t)$ даже при непрерывной $\varepsilon(t)$ вызывают разрывы $\sigma(t): \hat{\sigma}\left(t_{*}\right)=\eta \hat{\dot{\varepsilon}}\left(t_{*}\right), \hat{\dot{\sigma}}\left(t_{*}\right)=R(0+) \hat{\dot{\varepsilon}}\left(t_{*}\right)+\eta \hat{\ddot{\varepsilon}}\left(t_{*}\right)$. 
Следует отметить, что величина скачка процесса-отклика в любой точке $t_{*}$ линейно (и локально) зависит от величины скачка программь нагружения (и ее производных $в$ в этой же точке и не зависит от $t_{*} u$ от предыстории (если П $(t)$ и $\dot{R}(t)$ непрерывны), в частности, модуль величины скачка отклика не меняется при изменении знака скачка нагрузки. Эти свойства можно использовать как индикаторы применимости линейного ОС (1) (например, в испытаниях сплавов алюминия зафиксировано [21], что модуль величины скачка деформации вниз в момент сброса нагрузки меньше, чем величина скачка вверх в момент ее приложения).

На множестве непрерывных (и кусочно-дифференцируемых) при $t \geq 0$ функций (3) упрощается, и операторы (1) действуют по формуле:

$$
\sigma(t)=R(0) \varepsilon(t)+\int_{0}^{t} \dot{R}(t-\tau) \varepsilon(\tau) d \tau, \quad \varepsilon(t)=\Pi(0) \sigma(t)+\int_{0}^{t} \dot{\Pi}(t-\tau) \sigma(\tau) d \tau, t \geq 0 .
$$

Уравнением (1) задаются и все модели, собранные из линейных пружин и демпферов посредством последовательных и параллельных соединений (структурные реологические модели). Схемы и названия всех двух-, трех- и четырехзвенных моделей (в терминологии нет единства) приведены в [4] (п. 2, рис. 1). Можно доказать, что множество всех несократимых $n$-звенных моделей распадается ровно на два класса эквивалентности: РеМ- $n$ и СиМ- $n$ (структурно различные модели эквивалентны, если задаются одинаковыми семействами ФП или ФР). В частности $[1,6]$ :

1) эквивалентны трехзвенные РеМ Пойнтинга-Томсона и Кельвина ([4], рис. 1a);

2) все четыре РеМ-4 ([4], рис. 18) эквивалентны модели стандартного тела (последовательному соединению моделей Максвелла и Фойгта, то есть, РеМ-2 и СиМ-2);

3) все РеМ-2k эквивалентны параллельному соединению $k$ моделей Максвелла с разными временами релаксации;

4) все СиМ-2k эквивалентны последовательному соединению $k$ моделей Фойгта с разными временами ползучести (retardation time);

5) РеМ-(2k+1) получается из СиМ-2k последовательным присоединением упругого элемента, а СиМ- $(2 \mathrm{k}+1)$ - из РеМ-2k параллельным подключением вязкого элемента. Например, семейство ФП

$$
\Pi(t)=\alpha t+\beta-\gamma \mathrm{e}^{-\lambda t}, \quad \lambda>0, \alpha, \beta \geq 0, \gamma \in[0, \beta],
$$

порождает все РеМ-4 при $\gamma \in(0, \beta), \alpha, \beta>0$, а при $\alpha=0-$ РеМ-3. Так как П $(0)=$ $=\beta-\gamma$, то ФП (5) порождает СиМ, когда $\gamma=\beta$ : при $\alpha \beta=0-$ ньютоновскую жидкость, при $\alpha=0$ - модель Фойгта, при $\alpha>0$ получаются (все) СиМ-3. При $\gamma=0$ (5) дает модель Максвелла. Случай $\gamma<0$ приводит к нарушению ограничения $\ddot{\Pi}(t) \leq 0$, что вызывает возрастание кривой обратной ползучести (противоречие с данными испытаний материалов) $[1,2]$.

\section{2. Кривые обратной ползучести и требование убывания производной ФП}

«Ступеньку» $\sigma(t)=\bar{\sigma}[\mathrm{h}(t)-\mathrm{h}(t-T)]$, где $\bar{\sigma}>0, T>0$, оператор (1) переводит в процесс $\varepsilon(t)=\bar{\sigma} S(t, T), S(t, T)=\Pi(t) \mathrm{h}(t)-\Pi(t-T) \mathrm{h}(t-T), t \geq 0$. В точке $t=T$ функции $\varepsilon(t)$ и $\dot{\varepsilon}(t)$ имеют скачки $\hat{\varepsilon}(T)=-\bar{\sigma} \Pi(0), \hat{\dot{\varepsilon}}(T)=-\bar{\sigma} \dot{\Pi}(0)$, а на интервале $t>T$ уравнение КОП имеет вид:

$$
\varepsilon(t)=\bar{\sigma} S(t, T), \quad S(t, T)=\Pi(t)-\Pi(t-T), \quad t>T .
$$


КОП модели с ФП (5): $\varepsilon(t)=\bar{\sigma}\left(\alpha T+c \mathrm{e}^{-\lambda t}\right), c=\gamma\left(\mathrm{e}^{\lambda T}-1\right)$. У модели Максвелла $\gamma=0$ и $\varepsilon(t)=\bar{\sigma} \alpha T \equiv$ const при $t>T$, то есть вся накопленная за время $T$ деформация необратима. У моделей Фойгта и РеМ-3 $\alpha=0$, поэтому $\varepsilon(t)=\bar{\sigma} \gamma\left(\mathrm{e}^{\lambda T}-1\right) \mathrm{e}^{-\lambda t}$ и $\varepsilon(t) \rightarrow$ $\rightarrow 0$ при $t \rightarrow \infty$.

У всех стабильных материалов после снятия нагрузки наблюдается постепенное убывание (релаксация) деформации до некоторого уровня (нулевого для сетчатых полимеров в высокоэластичном состоянии). Это явление называется упругим восстановлением, возвратом, последействием, обратной ползучестью [11].

Из требования (нестрогого) убывания КОП (6) (с любым $T$ ) следует невозрастание $\dot{\Pi}(t)[1,2]$. Поэтому на ФП в ОС (1) следует налагать ограничение: П $(t)$ не имеет участков выпуклости вниз. Можно доказать, что ограничение $\ddot{\Pi}(t) \leq 0$ на ФП не является следствием остальных ограничений на ФР и ФП: существует гладкая убывающая $\Phi$ с с $\ddot{R}(t) \geq 0$, такая, что соответствующая ФП строго монотонна, но имеет участок с $\ddot{\Pi}(t)>0$ (хотя на всем этом участке $\ddot{R}(t)>0$ ) [1].

Итак, $\dot{\Pi}(t)$ убывает на луче $t>0$, значит, имеет предел $v=\dot{\Pi}(\infty) \geq 0$. Для моделей (5) $v=\alpha$, то есть $v>0$ при $\alpha>0$ (для РеМ-2, СиМ-3 и РеМ-4). Если ФП ограничена, то $v=0$ и $\varepsilon(t) \rightarrow 0$. Нетрудно доказать, что ФП всех моделей СиМ-2k и РеM-(2k+1) ограничены, а у РеМ-2k и СиМ-( $2 \mathrm{k}+1), k \in \mathbb{N}$, всегда $v>0$. Может быть $v=0$ и для неограниченной ФП, например, для П $(t)=\alpha t^{u}+\beta, u \in(0 ; 1), \alpha, \beta>0$. Ниже будет доказано, что именно от величины параметра $v(v=0$ или $v>0)$ зависит асимптотическое поведение КП для любых ступенчатых нагружений при $t \rightarrow \infty$ и накопление остаточной деформации.

Так как КОП (6) убывает и положительна (ограничена снизу), она имеет предел $\varepsilon_{\infty} \geq 0$ при $t \rightarrow \infty$, и $\varepsilon(\infty)=\bar{\sigma} S(\infty, T)$. По теореме Лагранжа $S(\infty, T)=v T$. Если $v>0$, память модели (точнее, память оператора П) не затухает, ибо след, оставленный прямоугольным импульсом нагрузки (остаточная деформация $\varepsilon_{\infty}=\bar{\sigma} v T$ ), не стирается никогда [6].

\section{3. Уравнение и общие свойства семейств КП при ступенчатом нагружении}

В силу (3) отклик для кусочно-постоянной программы нагружения с $n$ ступеньками $\sigma(t)=\sum_{i=1}^{n-1} \sigma_{i}\left[\mathrm{~h}\left(t-t_{i-1}\right)-\mathrm{h}\left(t-t_{i}\right)\right]+\sigma_{n} \mathrm{~h}\left(t-t_{n-1}\right)$ (полагаем, что $t_{0}=0$ и $t_{i}>$ $\left.>t_{i-1}\right)$ имеет вид:

$$
\varepsilon(t)=\sum_{i=1}^{n-1} \sigma_{i} S\left(t-t_{i-1}, t_{i}-t_{i-1}\right)+\sigma_{n} \Pi\left(t-t_{n-1}\right) \mathrm{h}\left(t-t_{n-1}\right), \quad t>0,
$$

или

$$
\begin{gathered}
\varepsilon(t)=\sum_{i=0}^{n-1} \hat{\sigma}_{i} \Pi\left(t-t_{i}\right) \mathrm{h}\left(t-t_{i}\right), t>0, \\
\text { то есть } \varepsilon(t)=\sigma_{1} \Pi(t)+\sum_{k=1}^{i} \hat{\sigma}_{k} \Pi\left(t-t_{k}\right), t \in\left(t_{i} ; t_{i+1}\right),
\end{gathered}
$$

где $\hat{\sigma}_{i}=\sigma_{i+1}-\sigma_{i}$ сккачки напряжения в точках $t_{i}, \sigma_{0}=0$. Скачки $\varepsilon(t)$ и $\dot{\varepsilon}(t)$ в точке $t_{i}$ :

$$
\hat{\varepsilon}_{i}=\hat{\sigma}_{i} \Pi(0), \quad \hat{\dot{\varepsilon}}_{i}=\Pi(0) \hat{\dot{\sigma}}_{i}+\dot{\Pi}(0) \hat{\sigma}_{i}=\dot{\Pi}(0) \hat{\sigma}_{i},
$$

так как $\hat{\dot{\sigma}}_{i}=0$ в данном случае. 
При $t \rightarrow \infty$ из (7) следеут:

$$
\varepsilon(t)=\sigma_{n} \Pi\left(t-t_{n-1}\right)+s_{+}+o(1)
$$

где

$$
s_{+}=v \sum_{i=1}^{n-1} \sigma_{i}\left(t_{i}-t_{i-1}\right), \quad v=\dot{\Pi}(\infty) .
$$

Если $\sigma_{n}=0$, то $\varepsilon(t) \rightarrow s_{+}$при $t \rightarrow \infty$, то есть КП (7) имеет горизонтальную асимптоту.

Если $\sigma_{n} \neq 0$, но $v=0$, то $s_{+}=0$, и потому при $t \rightarrow \infty$ имеем: $\varepsilon(t)-\sigma_{n} \Pi\left(t-t_{n-1}\right) \rightarrow$ $\rightarrow 0, \varepsilon(t)-\sigma_{n} \Pi(t) \rightarrow 0, \Pi(t)-\Pi\left(t-t_{n-1}\right) \rightarrow 0$. В частности, $v=0$, если $\Pi(t)$ ограничена (например, все СиМ-2k и РеМ-(2k+1)); в этом случае $s_{+}=0$ и $\varepsilon(t) \rightarrow \sigma_{n} \Pi_{\infty}$ при $t \rightarrow \infty$. Если П $(t)$ не ограничена и $\sigma_{n} \neq 0$, то $\varepsilon(t) \sim \sigma_{n} \Pi\left(t-t_{n-1}\right)$ и $|\varepsilon(t)|$ неограниченно возрастает.

Если $v \neq 0$ (как у РеМ-2k и СиМ-(2k+1), $k \in \mathbb{N}$ ) и $\sigma_{n} \neq 0$, то $\varepsilon(t) \rightarrow \infty$ при $t \rightarrow \infty$, $\sigma_{n}\left(\Pi(t)-\Pi\left(t-t_{n-1}\right)\right) \rightarrow \sigma_{n} v t_{n-1} \neq 0, \varepsilon(t)-\sigma_{n} \Pi\left(t-t_{n-1}\right) \rightarrow s_{+}$(значение $s_{+}$может быть любым, но если все $\sigma_{i} \geq 0$ и хотя бы одно $\sigma_{k}>0$, то и $\left.s_{+}>0\right)$, а $\varepsilon(t)-\sigma_{n} \Pi(t)$, вообще говоря, не стремится к нулю при $t \rightarrow \infty$. Если у ФП есть наклонная асимптота, то есть существует

$$
\lim _{t \rightarrow \infty}(\Pi(t)-v t)=b,
$$

то КП (7) имеет асимптоту $\varepsilon=\sigma_{n}\left[v\left(t-t_{n-1}\right)+b\right]+s_{+}$(РеМ-4). Отметим, что если все $\sigma_{i} \geq 0$ для $i<n$, то отклонение $\varepsilon(t)-\sigma_{n} \Pi\left(t-t_{n-1}\right)$ убывает на луче $t>t_{n-1}$, так как все слагаемые в (7), кроме последнего, убывают по $t$ при $t>t_{i}$.

Для иллюстрации рассмотрим случай $n=2$. Тогда при $t>t_{1}$ имеем:

$$
\begin{aligned}
\varepsilon(t)=\sigma_{1} \Pi(t)+ & \left(\sigma_{2}-\sigma_{1}\right) \Pi\left(t-t_{1}\right)=\sigma_{1} S\left(t, t_{1}\right)+\sigma_{2} \Pi\left(t-t_{1}\right)= \\
& =\sigma_{2} \Pi(t)+\left(\sigma_{1}-\sigma_{2}\right) S\left(t, t_{1}\right),
\end{aligned}
$$

$S\left(t, t_{1}\right)=\Pi(t)-\Pi\left(t-t_{1}\right)$. Для КП (9) с $\sigma_{1}>0$ всегда $\varepsilon(t)>\sigma_{2} \Pi\left(t-t_{1}\right)$ и $\varepsilon(t)-\sigma_{2} \Pi\left(t-t_{1}\right)$ убывает; $\varepsilon(t)>\sigma_{2} \Pi(t)$ при $\sigma_{2}<\sigma_{1}$ и $\sigma_{2} \Pi\left(t-t_{1}\right)<\varepsilon(t)<\sigma_{2} \Pi(t)$ при $\sigma_{2}>\sigma_{1}$ (рис. 1).

Так как $S\left(t, t_{1}\right) \rightarrow v t_{1} \geq 0$ при $t \rightarrow \infty$ и $S_{+}=v \sigma_{1} t_{1}$, то КП (9) имеет асимптотику

$$
\begin{aligned}
& \varepsilon(t)=\sigma_{2} \Pi\left(t-t_{1}\right)+\sigma_{1} v t_{1}+o(1), \quad t \rightarrow \infty, \\
& \text { или } \varepsilon(t)=\sigma_{2} \Pi(t)+\left(\sigma_{1}-\sigma_{2}\right) v t_{1}+o(1) .
\end{aligned}
$$

Если $v=0$, то при $t \rightarrow \infty: \Pi(t)-\Pi\left(t-t_{1}\right) \rightarrow 0, \varepsilon(t)-\sigma_{2} \Pi\left(t-t_{1}\right) \rightarrow 0$ и $\varepsilon(t)-$ $-\sigma_{2} \Pi(t) \rightarrow 0$, то есть (9) стремится к КП для уровня напряжения $\sigma_{2}$ (модель забывает про нагрузку при $\left.t<t_{1}\right)$. Если ФП ограничена, то $v=0$ и $\varepsilon(\infty)=\sigma_{2} \Pi(\infty)$, то есть КП имеет горизонтальную асимптоту.

Если $v \neq 0$ (тогда $\Pi(\infty)=\infty$ ) и $\sigma_{2} \neq 0$, то при $t \rightarrow \infty: \varepsilon \rightarrow \infty, \varepsilon(t)-\sigma_{2} \Pi\left(t-t_{1}\right) \rightarrow$ $\rightarrow s_{+}$, где $s_{+}=v \sigma_{1} t_{1}\left(s_{+}>0\right.$ при $\left.\sigma_{1}>0\right), \varepsilon(t)-\sigma_{2} \Pi(t) \rightarrow v\left(\sigma_{1}-\sigma_{2}\right) t_{1} \neq 0$, то есть оба отклонения не стремятся к нулю при $t \rightarrow \infty$, и П $(t)-\Pi\left(t-t_{1}\right) \rightarrow v t_{1} \neq 0$. Например, у модели Максвелла $(\Pi=\alpha t+\beta) v=\alpha \neq 0$, КП (9): $\varepsilon(t)=\sigma_{2}\left[\alpha\left(t-t_{1}\right)+\beta\right]+\alpha \sigma_{1} t_{1}=$ $=\sigma_{2}[\alpha t+\beta]+\left(\sigma_{1}-\sigma_{2}\right) \alpha t_{1}$. Эта КП линейна по $t$, возрастает при $\sigma_{2}>0$, отличается от $\sigma_{2} \Pi\left(t-t_{1}\right)$ на постоянную $s_{+}=\alpha \sigma_{1} t_{1}$, а от $\sigma_{2} \Pi(t)-$ на $\left(\sigma_{1}-\sigma_{2}\right) \alpha t_{1}$. Модель Максвелла (как и все РеM-2k и СиМ-(2k+1), $k \in \mathbb{N})$ ) бесконечно долго помнит о нагрузке $\sigma_{1}$ при $t<t_{1}$. 


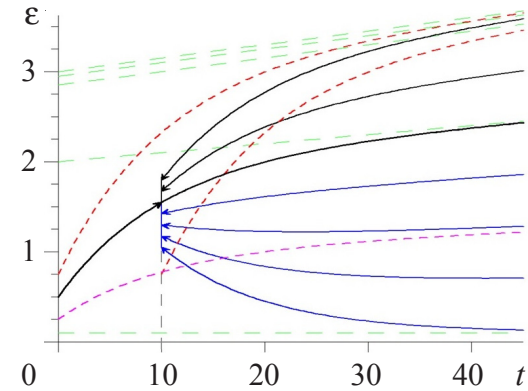

a)

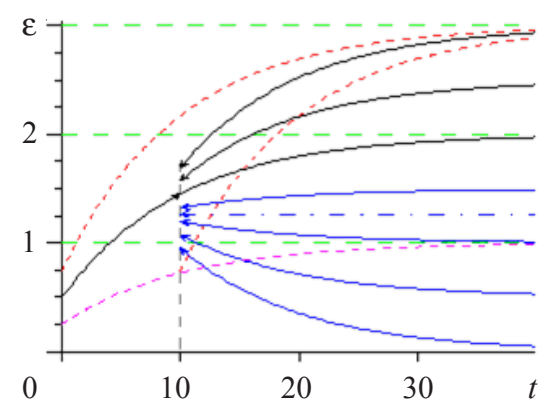

в)

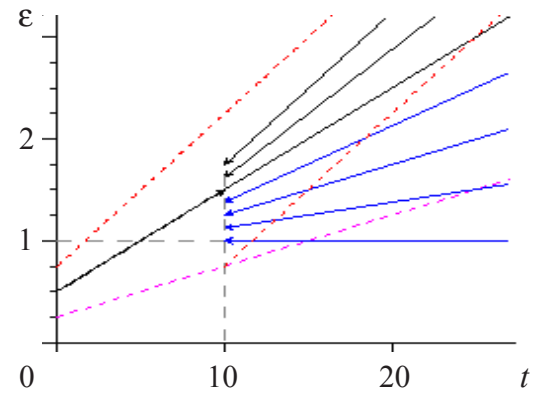

б)

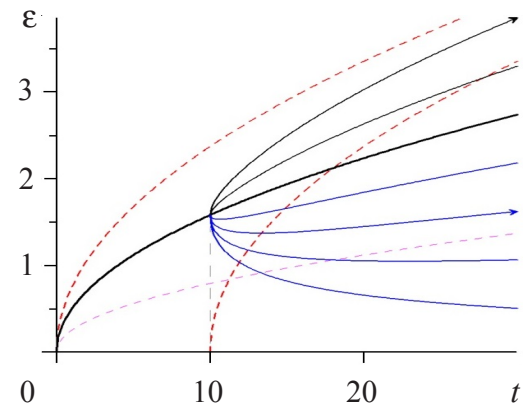

2)

Рис. 1

На рис. 1 прииведены КП РеМ-4 (П = $\left.\alpha t+\beta-\gamma \mathrm{e}^{-\lambda t}\right)$ с $\alpha=0,01, \beta=2, \gamma=1,5$ $\lambda=0,1$ для $t_{1}=10, \sigma_{1}=1, \sigma_{2}=1 ; 1,25 ; 1,5$ (черные КП для $\sigma_{2} \geq \sigma_{1}$ ) и $\sigma_{2}=0,75 ; 0,5$; 0,$25 ; 0$ (синие КП для $\sigma_{2}<\sigma_{1}$ ). Так как П $(0)=\beta-\gamma \neq 0$, то в точке $t=t_{1}$ КП имеют разрыв первого рода. Для РеМ-4 $v=\alpha \neq 0, s_{+}=\alpha \sigma_{1} t_{1}$; при $t \rightarrow \infty$ КП имеют асимптоту $\varepsilon=\sigma_{2}\left[\alpha\left(t-t_{1}\right)+\beta\right]+\alpha \sigma_{1} t_{1}, \sigma_{2}\left(\Pi(t)-\Pi\left(t-t_{1}\right)\right) \rightarrow \sigma_{2} \alpha t_{1} \neq 0$ и $\varepsilon(t)-$ $-\sigma_{2}\left(\Pi\left(t-t_{1}\right) \rightarrow \sigma_{1} \alpha t_{1} \neq 0\right.$. Красные штриховые линии - обычная КП $\varepsilon=\sigma_{2} \Pi(t)$ и ее сдвиг $\varepsilon=\sigma_{2} \Pi\left(t-t_{1}\right)$ для $\sigma_{2}=1,5$, зеленые прямые - их асимптоты. Розовая штриховая линия - КП $\varepsilon=\sigma_{2} \Pi(t)$ для $\sigma_{2}=0,5$.

На рис. 16 приведены КП модели Максвелла с $\alpha=0,1, \beta=0,5$ для $t_{1}=10, \sigma_{1}=1$ и тех же $\sigma_{2}$. Красные штриховые прямые - обычная КП $\varepsilon=\sigma_{2} \Pi(t), \varepsilon=\sigma_{2} \Pi\left(t-t_{1}\right)$ для $\sigma_{2}=1,5$.

На рис. 1 в приведены КП для РеМ-3: П $(t)=\beta-\gamma \mathrm{e}^{-\lambda t}, \gamma \in(0 ; \beta)$ с $\beta=2, \gamma=1,5$, $\lambda=0,1$ для $t_{1}=10, \sigma_{1}=1$ и тех же значений $\sigma_{2}$. Для РеМ-3 $v=0$ и потому при $t \rightarrow \infty$ : $\sigma_{2}\left(\Pi(t)-\Pi\left(t-t_{1}\right)\right) \rightarrow 0, \varepsilon(t)-\sigma_{2} \Pi(t) \rightarrow 0$ и каждая КП имеет горизонтальную асимптоту $\varepsilon=\sigma_{2} \beta$. КП модели Фойгта (СиМ-2, $\gamma=\beta$ ) отличаются от КП РеМ-3 лишь тем, что П $(0)=0$ и в точке $t=t_{1}$ все КП непрерывны.

На рис. 12 приведены КП для ФП П $(t)=A t^{u} \mathrm{c} A=0,5, u=0,5$ для $t_{1}=10, \sigma_{1}=1$ и тех же значений $\sigma_{2}$. Так как П $(0)=0$, то в точке $t=t_{1}$ все КП не имеют разрыва. Так как $\dot{\Pi}(0)=\infty$, то у всех КП Пं $\left(t_{1}+0\right)= \pm \infty$ (знак совпадает с $\left.\operatorname{sgn}\left(\sigma_{2}-\sigma_{1}\right)\right)$. Для любого $u<1$, очевидно, $v=0$, и потому $\sigma_{2}\left(\Pi(t)-\Pi\left(t-t_{1}\right)\right) \rightarrow 0$ и $\varepsilon(t)-\sigma_{2} \Pi(t) \rightarrow 0$ при $t \rightarrow \infty$ (точнее, $\left.\varepsilon(t)-\sigma_{2} \Pi(t)=\left(\sigma_{1}-\sigma_{2}\right)\left[u A t_{1} t^{u-1}+O\left(t^{u-2}\right)\right]\right)$. 


\section{4. О существовании точки минимума у кривых ползучести с неполной разгрузкой}

При фиксированных $\sigma_{1}$ и $t_{1}$ семейство (9) - пучок кривых, непрерывно зависящий от параметра $\sigma_{2} \in \mathbb{R}$. При $\sigma_{2}=\sigma_{1}$ получаем обычную возрастающую КП, а при $\sigma_{2}=0$ - убывающую КОП. Возникает вопрос: как ведут себя КП (9) при $\sigma_{2} \in\left(0, \sigma_{1}\right)$ и при каком отношении $\sigma_{2} / \sigma_{1}>0$ происходит смена возрастания КП на убывание? Точнее: когда у КП появляется интервал убывания и точка минимума (см. рис. 12)? Этот эффект наблюдается в экспериментах $[11,16,21,22]$.$) Из (10) следует, что в$ случае П $(\infty)=\infty$ интервал убывания КП при $\sigma_{2}>0$ обязательно конечен, так как ее главная часть возрастает (если $\Pi(\infty)<\infty, \varepsilon(t)=\sigma_{2} \Pi(\infty)+o(1)$ и КП может убывать на всем луче $\left.t \geq t_{1}\right)$,

$$
\dot{\varepsilon}(t)=\sigma_{1} \dot{\Pi}(t)-\left(\sigma_{1}-\sigma_{2}\right) \dot{\Pi}\left(t-t_{1}\right)=\sigma_{1} \dot{\Pi}\left(t-t_{1}\right)\left[Q\left(t, t_{1}\right)-\left(\sigma_{1}-\sigma_{2}\right) / \sigma_{1}\right],
$$

где

$$
Q\left(t, t_{1}\right)=\dot{\Pi}(t) / \dot{\Pi}\left(t-t_{1}\right), \quad t>t_{1}>0 .
$$

Из (11) следует условие экстремума $\varepsilon(t)$ :

$$
Q\left(t, t_{1}\right)=1-\sigma_{2} / \sigma_{1} .
$$

Таким образом, поведение КП (9), положение точек экстремума и их зависимость от параметров модели и программы нагружения определяются свойствами функции $Q\left(t, t_{1}\right)$ (ее множеством значений и интервалами монотонности).

Так как $\dot{\Pi}(t)>0$ и не возрастает, то $0<Q\left(t, t_{1}\right)<1\left(\sup Q\left(t, t_{1}\right)<1\right.$ равносильно убыванию Пं( $t))$. При $t \rightarrow \infty$ из (11) следует $\dot{\varepsilon}(t) \rightarrow \sigma_{2} v$, а $Q\left(t, t_{1}\right) \rightarrow 1$, если $v \neq 0$ (если $v=0$, возможно $Q(\infty)<1)$. Предел в точке $t_{1}$

$$
q\left(t_{1}\right)=Q\left(t_{1}+0, t_{1}\right)=\dot{\Pi}\left(t_{1}\right) / \dot{\Pi}(+0)
$$

может принимать все значения из отрезка $[0,1]$ : если $\dot{\Pi}(0)=\infty$, то $q\left(t_{1}\right) \equiv 0$ (и $\left.\dot{\varepsilon}\left(t_{1}+0\right)=-\infty\right)$; если $\dot{\Pi}(t) \neq$ const, то $q<1$. Для модели Максвелла $Q\left(t, t_{1}\right) \equiv 1$, $q\left(t_{1}\right) \equiv 1$, КП (9) возрастает при всех $\sigma_{2}>0$ (см. рис. 16$)$.

Для РеМ-3 и СиМ-2 $\left(\Pi(t)=\beta-\gamma \mathrm{e}^{-\lambda t}, \gamma \in(0 ; \beta]\right)$ будет $Q(t)=\mathrm{e}^{-\lambda t_{1}}=\mathrm{const}=$ $=q\left(t_{1}\right) \in(0,1)$, уравнение (12) имеет решение лишь при одном значении $\sigma_{2}=\sigma_{*}$, $\sigma_{*}=\sigma_{1}\left(1-q\left(t_{1}\right)\right)$, и для него (11) дает $\dot{\varepsilon}(t) \equiv 0$ для РеМ-3, или $\varepsilon(t)=$ const. Из (11) следует, что при $\sigma_{2}>\sigma_{*}$ будет $\dot{\varepsilon}(t)>0$ при всех $t>t_{1}$, а при $\sigma_{2}<\sigma_{*}$ будет $\dot{\varepsilon}(t)<0$. Для РеМ-3 смена возрастания КП на убывание (а также выпуклости) происходит тотально на всем луче $t \geq t_{1}$, синфазно во всех точках. Синяя штрихпунктирная прямая на рис. $18-$ КП при $\sigma_{2}=\sigma_{*}$ (для $\lambda=0,1$ и $t_{1}=10$ имеем $q=\mathrm{e}^{-\lambda t_{1}}=\mathrm{e}^{-1} \approx 0,37$, $\left.\sigma_{*}=\sigma_{1}(1-q) \approx 0,63 \sigma_{1}, \varepsilon\left(t, \sigma_{*}\right) \equiv \varepsilon(\infty)=\sigma_{*} \beta\right)$.

Для ФП П $(t)=c+A t^{u}, u \in(0,1), A>0$, имеем: $Q\left(t, t_{1}\right)=\left(1-t_{1} / t\right)^{1-u}$ (не зависит от $A$ и $c)$. $Q\left(t, t_{1}\right)$ возрастает по $t, q=0, Q(\infty)=1$ (две голубые кривые на рис. $2 a-$ это $Q(t)$ для $t_{1}=5$ : верхняя $-u=0,5$, нижняя $\left.-u=0,001\right)$. Поэтому уравнение (12) имеет только один корень $t_{*}=t_{*}\left(\sigma_{2}\right)>t_{1}$ при любом $\sigma_{2} \in\left(0, \sigma_{1}\right)$; из $(11)$ следует, что $t_{*}$ - точка минимума КП (9). Согласно (12) $t_{*}=t_{*}\left(\sigma_{2}\right)$ убывает (так как $Q(t)$ возрастает), $t_{*}(0+)=\infty, t_{*}\left(\sigma_{1}-0\right)=t_{1}$ (точка минимума КП $t_{*}$ двигается вправо от $t_{1}$ до $\infty$ с изменением $\sigma_{2}$ от $\sigma_{1}$ до нуля). Для этой ФП $v=0$ при любом $u<1$, поэтому $\varepsilon(t)-\sigma_{2} \Pi(t) \rightarrow 0$ при $t \rightarrow \infty$. На рис. 12 синим цветом показаны КП для ФП с $A=$ $=0,25, u=0,5, c=0$ (тогда П(0)=0 и КП непрерывны), $t_{1}=10, \sigma_{1}=1, \sigma_{2}=0,75 ; 0,5$; 
0,25; 0 (нижняя кривая - КОП при $\sigma_{2}=0$ ). Так как Пं(0)= , то $\dot{\varepsilon}\left(t_{1}+0\right)=-\infty$ при любом $\sigma_{2}<\sigma_{1}$. Розовая штриховая КП - обычная КП при $\sigma=0,5$.

Вернемся к случаю произвольной ФП. В точке $t_{1}$

$$
\dot{\varepsilon}\left(t_{1}+0\right)=\sigma_{1} \dot{\Pi}\left(t_{1}\right)-\left(\sigma_{1}-\sigma_{2}\right) \dot{\Pi}(+0)
$$

и $\dot{\varepsilon}\left(t_{1}+0\right)=0$ равносильно $\sigma_{2}=\sigma_{*}=\sigma_{1}\left(1-q\left(t_{1}\right)\right)$. При $\sigma_{2}>\sigma_{*}$ будет $\dot{\varepsilon}\left(t_{1}\right)>0$, а при $\sigma_{2}<\sigma_{*}$ будет $\dot{\varepsilon}\left(t_{1}\right)<0$, следовательно, $\varepsilon(t)<\varepsilon\left(t_{1}\right)$ в правой окрестности $t_{1}$. При $\sigma_{2}=$ $=\sigma_{*}(11)$ принимает вид: $\dot{\varepsilon}(t)=\sigma_{1} \dot{\Pi}\left(t-t_{1}\right)\left[Q\left(t, t_{1}\right)-q\left(t_{1}\right)\right]$, поэтому знак $\dot{\varepsilon}(t)$ совпадает со знаком $Q\left(t, t_{1}\right)-q\left(t_{1}\right)$. Так как $\dot{Q}=\left[\ddot{\Pi}(t) \dot{\Pi}\left(t-t_{1}\right)-\dot{\Pi}(t) \ddot{\Pi}\left(t-t_{1)}\right]\left[\dot{\Pi}\left(t-t_{1}\right)\right]^{-2}\right.$ и $\dot{\Pi}>0$, то $\dot{Q}(t) \geq 0$ при всех $t>t_{1}>0$ тогда и только тогда, когда $\ddot{\Pi}(t) / \dot{\Pi}(t) \geq$ $\geq \ddot{\Pi}\left(t-t_{1}\right) / \dot{\Pi}\left(t-t_{1}\right), t \geq t_{1}$,то есть производная по времени функции $\ln \dot{\Pi}(t)$ не убывает при $t>0$. С помощью этого критерия легко доказывается возрастание $Q(t)$ для ФП (5) с $\alpha>0$ (то есть РеМ-4 и СиМ-3) и ФП П $(t)=A \ln (\lambda t+\tau)+c, \tau \geq 1, \lambda>0$.

Однако возрастание $Q(t)$ не вытекает автоматически из наложенных на ФП ограничений: $Q(t)$ может иметь интервал убывания. Например, для ФП П $(t)=$ $=A \operatorname{arctg} \lambda t+c, A, \lambda, c>0$, имеем: $Q(t)=1-t_{1}\left(2 t-t_{1}\right)\left(\lambda^{-2}+t^{2}\right)^{-1}$ или $Q(x)=1-$ $-(2 x-1)\left(x^{2}+\mu\right)^{-1}$, где $x=t t_{1}^{-1}>1, \mu=\left(\lambda t_{1}\right)^{-2}>0, Q^{\prime}(x)=0$ в точке $x=x_{*}, x_{*}=0,5+$ $+0,5(1+4 \mu)^{0,5}>1$. Точка минимума $x_{*}$ возрастает по $\mu$ и убывает с ростом $\lambda t_{1}$, при $\lambda t_{1} \rightarrow \infty x_{*} \rightarrow 1$, а при $\lambda t_{1} \rightarrow 0 x_{*} \rightarrow \infty$. Итак, для любых $A, \lambda, c>0$ и $t_{1}>0$ функция $Q(t)$ имеет одну точку минимума $t_{m}=x_{*} t_{1}$, убывает на интервале $\left(t_{1}, t_{m}\right)$ и возрастает на интервале $\left(t_{m}, \infty\right)$. При этом $Q(\infty)=1, q=\left(1+\left(\lambda t_{1}\right)^{2}\right)^{-1}, t_{m}(\lambda)$ убывает, при $\lambda \rightarrow \infty$ $t_{m} \rightarrow t_{1}$, а при $\lambda \rightarrow 0 t_{m} \rightarrow \infty$. Три красные кривые на рис. $2 a-$ графики $Q(t)$ для $t_{1}=5$ и $\lambda=0,05 ; 0,1 ; 0,5$. Черные (возрастающие) кривые - графики $Q(t)$ для ФП (5) (то есть РеМ-4 и СиМ-3): $Q(t)=\left(\alpha+\gamma \lambda \mathrm{e}^{-\lambda t}\right) /\left(\alpha+\gamma \lambda \mathrm{e}^{\lambda t_{1}} \mathrm{e}^{-\lambda t}\right)$ с $\alpha=0,001, \gamma=1,5, \lambda=0,1$; 0,$2 ; 0,3$, а штриховая кривая - для $\alpha=10^{-8}(\alpha \approx 0), \lambda=0,3$.

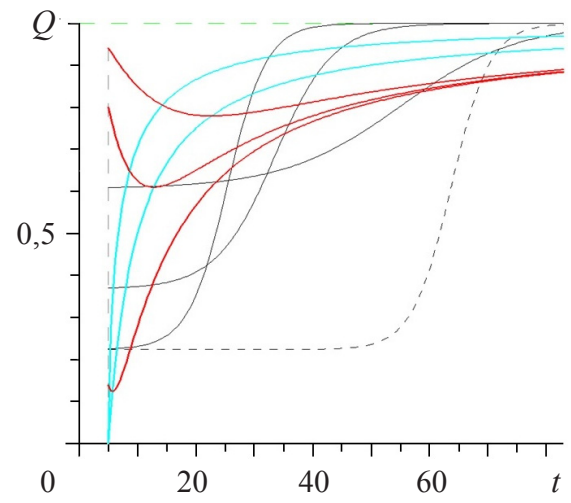

a)

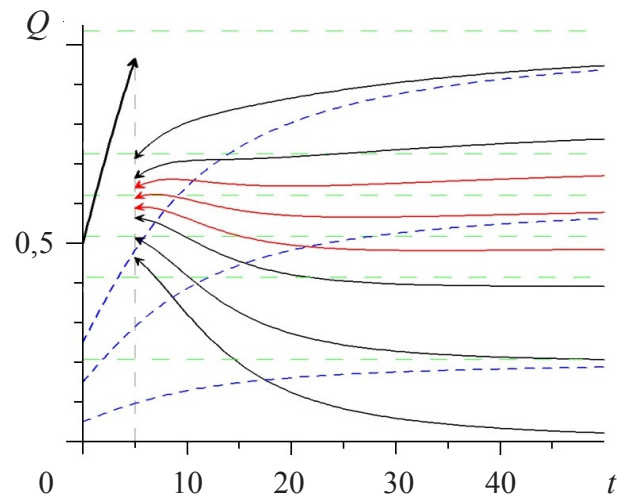

б)

Рис. 2

Итак, если $Q\left(t, t_{1}\right)$ возрастает по $t$ на всем луче $t \geq t_{1}$, то уравнение (12) имеет ровно один корень $t_{*}=t_{*}\left(\sigma_{2}\right)>t_{1}$, когда (и только тогда) $1-\sigma_{2} / \sigma_{1} \in\left(q\left(t_{1}\right), Q\left(\infty, t_{1}\right)\right)$; при этом $t_{*}$ - точка минимума КП (9) (из (11) следует, что $\dot{\varepsilon}<0$ при $t \in\left(t_{1}, t_{*}\right)$ ). С уменьшением $\sigma_{2}$ правая часть уравнения (12) растет и потому $t_{*}\left(\sigma_{2}\right)$ монотонно сдвигается вправо $\left(t_{*} \rightarrow \infty\right.$ при $\left.\sigma_{2} \rightarrow 0\right)$. Поэтому корень $t_{*}$ уравнения (12) появляется на полуинтервале $\left[t_{1}, \infty\right)$ именно в точке $t_{1}: t_{*}=t_{1}$ при $\sigma_{2}=\sigma_{*}=\sigma_{1}(1-q)$. Таким 
образом, КП (9) при $\sigma_{2} \geq \sigma_{*}$ возрастает на всем луче $\left[t_{1}, \infty\right)$, а при $\sigma_{2}<\sigma_{*}$ она сначала убывает на интервале $\left[t_{1}, t_{*}\right)$, а затем возрастает при $t>t_{*}$ (см. рис. $1 a$, ). При $t \rightarrow \infty$ КП (9) всегда имеет асимптотику (10).

Если же $Q(t)$ убывает при $t \in\left[t_{1}, t_{m}\right]$, а затем возрастает (см. рис. $2 a$ ), то уравнение (12) либо не имеет корней при $1-\sigma_{2} / \sigma_{1}<\min Q(t)=Q\left(t_{m}\right)$ (при таких $\sigma_{2}$ КП (9) возрастает на всем интервале $\left.\left[t_{1},+\infty\right)\right)$, либо имеет два корня $\widetilde{t}\left(\sigma_{2}\right)<t_{*}\left(\sigma_{2}\right)$ при $Q\left(t_{m}\right)<1-\sigma_{2} / \sigma_{1}<q$ (при таких $\sigma_{2}$ КП (9) возрастает на $\left(t_{1}, \widetilde{t}\right)$ и $\left(t_{*}, \infty\right)$ и убывает на $\left.\left[\widetilde{t}, t_{*}\right]\right)$, либо имеет один корень, когда $q<1-\sigma_{2} / \sigma_{1}<Q(\infty)$. С уменьшением $\sigma_{2}$ правая часть (12) растет, при $\sigma_{2} / \sigma_{1}=1-Q\left(t_{m}\right)$ в точке $t_{m}$ появляются корни $\tilde{t}=t_{*}$ и начинают расходиться в стороны, затем точка максимума КП $\tilde{t}\left(\sigma_{2}\right)$ достигает точки $t_{1}$ при $\sigma_{2}=\sigma_{*}=\sigma_{1}(1-q)$ и исчезает интервал возрастания $[\widetilde{t}, t]$, а точка минимума $t_{*}\left(\sigma_{2}\right)$ продолжает двигаться вправо (при $\sigma_{2} \rightarrow 0 t_{*} \rightarrow \infty$ ).

Эти общие свойства КП (9) иллюстрирует рис. 2б: на нем приведены КП для ФП П $=A \operatorname{arctg} \lambda t+c, A=1, c=0,5, \lambda=0,1$ при $t_{1}=5, \sigma_{1}=1, \sigma_{2}=0 ; 0,1 ; 0,2 ; 0,25$; 0,$3 ; 0,35 ; 0,4 ; 0,5$. Зеленые прямые - их горизонтальные асимптоты $\varepsilon=0,5(\pi+1) \sigma_{2}$ (любая КП (9) при $\sigma_{2} \in\left(0, \sigma_{1}\right)$ стремится к своей асимптоте снизу). На КП с $\sigma_{2}=$ $=0,25 ; 0,3 ; 0,35$ (красные КП) имеются две точки экстремума, на КП с $\sigma_{2} \in(0 ; 0,2]$ есть только точка минимума, КП с $\sigma_{2}=0$ (кривая обратной ползучести) монотонно убывает на луче $t \geq t_{1}$ и имеет асимптоту $\varepsilon=0$; КП для $\sigma_{2} \geq 0,4$ монотонно возрастают. Синие штриховые кривые - обычные КП $\varepsilon=\sigma_{2} \Pi(t)$ для $\sigma_{2}=0,1 ; 0,3 ; 0,5$.

Таким образом, исследование общих свойств функции $Q\left(t, t_{1}\right)$, соответствующей произвольной ФП, позволило классифицировать возможные типы поведения кривых ползучести при двухступенчатом нагружении с $\sigma_{2} \in\left(0, \sigma_{1}\right)$ (подробнее см. $[1,6])$ и указать, когда линейное ОС (1) способно описывать наличие точки минимума у КП.

\section{5. Некоммутативность при двухступенчатом нагружении, асимптотическая коммутативность}

Одквистом [10] было подмечено (а затем проверялось в экспериментах), что для теории ползучести с упрочнением вида $\dot{\varepsilon}=g(\varepsilon) f(\sigma)$ справедливо свойство коммутативности при двухступенчатом нагружении [11]: для двух программ нагружения, отличающихся лишь порядком (мгновенного) приложения напряжений $\sigma_{1}$ и $\sigma_{2}$ в течение одинакового времени $T$, деформация в момент $t=2 T$ будет одна и та же: $\varepsilon_{12}(2 T)=\varepsilon_{21}(2 T)$. В испытаниях материалов это свойство не выполняется, а наблюдается $\varepsilon_{12}(2 T)>\varepsilon_{21}(2 T)$ для $\sigma_{2}>\sigma_{1}[11,15]$. Докажем, что линейное ОС (1) качественно верно описывает экспериментальные факты и всегда дает $\varepsilon_{12}(2 T)>\varepsilon_{21}(2 T)$.

Для входных процессов $\sigma(t)=\sigma_{1} \mathrm{~h}(t)+\left(\sigma_{2}-\sigma_{1}\right) \mathrm{h}(t-T), \sigma(t)=\sigma_{2} \mathrm{~h}(t)+\left(\sigma_{1}-\right.$ $\left.-\sigma_{2}\right) \mathrm{h}(t-T)$ отклики при $t>T$ определяются по формуле (9) с $n=2, t_{1}=T$ :

$$
\begin{gathered}
\varepsilon_{12}(t)=\sigma_{1} \Pi(t)+\left(\sigma_{2}-\sigma_{1}\right) \Pi(t-T), \quad \varepsilon_{21}(t)=\sigma_{2} \Pi(t)+\left(\sigma_{1}-\sigma_{2}\right) \Pi(t-T), \\
\varepsilon_{12}(t)-\varepsilon_{21}(t)=\left(\sigma_{2}-\sigma_{1}\right)[2 \Pi(t-T)-\Pi(t)], \quad t>T .
\end{gathered}
$$

В частности: $\varepsilon_{12}(2 T)-\varepsilon_{21}(2 T)=\left(\sigma_{2}-\sigma_{1}\right)[2 \Pi(T)-\Pi(2 T)]$. Так как $2 \Pi(t)-$ - П $(2 t)-\Pi(0) \geq 0$ в силу выпуклости вверх ФП, то для любых двух программ нагружения с $\sigma_{2}>\sigma_{1}$ ОС (1) всегда дает $\varepsilon_{12}(2 T)>\varepsilon_{21}(2 T)$. 
Равенство $\varepsilon_{12}(2 T)-\varepsilon_{21}(2 T)=0$ возможно лишь в случае одновременного выполнения двух условий: $\Pi(0)=0$ и П $(t)$ линейна на $[0,2 T]$, то есть возможно только для вязкого элемента (с ФП П = $\alpha t)$. Для модели Максвелла $2 П(T)-\Pi(2 T)$ $=\beta=1 / E$ и $\varepsilon_{12}(2 T)-\varepsilon_{21}(2 T)==\left(\sigma_{2}-\sigma_{1}\right) \beta$.

Рассмотрим более тонкий вопрос о связи между откликами на две трехступенчатые программы нагружения с напряжением $\bar{\sigma}=\left(\sigma_{1}+\sigma_{2}\right) / 2$ на интервале $t>t_{2}=$ $=2 T$ (как и ранее, предполагаем, что $\sigma_{2}>\sigma_{1}$ ). Докажем, что для откликов верно неравенство $\varepsilon_{12}(t, \bar{\sigma})>\varepsilon(t, \bar{\sigma})>\varepsilon_{21}(t, \bar{\sigma})$ при всех $t>2 T$, где $\varepsilon(t, \bar{\sigma})=\bar{\sigma} \Pi(t)-$ обычная КП, причем при $t \rightarrow \infty$ для любой ФП $\varepsilon_{12}(t, \bar{\sigma})-\varepsilon_{21}(t, \bar{\sigma}) \rightarrow 0$ (асимптотическая коммутативность).

По формуле (8), учитывая, что скачки напряжения в точках $t_{1}=T$ и $t_{2}=2 T$ для двух программ нагружения различаются лишь знаком, получим:

$$
\begin{gathered}
\varepsilon_{12}(t, \bar{\sigma})=\sigma_{1} \Pi(t)+\left(\sigma_{2}-\sigma_{1}\right) \Pi(t-T)-0,5\left(\sigma_{2}-\sigma_{1}\right) \Pi(t-2 T) \text { при } t>2 T, \\
\varepsilon_{21}(t, \bar{\sigma})=\sigma_{2} \Pi(t)-\left(\sigma_{2}-\sigma_{1}\right) \Pi(t-T)+0,5\left(\sigma_{2}-\sigma_{1}\right) \Pi(t-2 T) .
\end{gathered}
$$

Отсюда следует

$$
\begin{gathered}
\varepsilon_{12}(t)-\bar{\sigma} \Pi(t)=0,5\left(\sigma_{2}-\sigma_{1}\right) \Delta(t, T)=\bar{\sigma} \Pi(t)-\varepsilon_{21}(t), \\
\varepsilon_{12}(t)-\varepsilon_{21}(t)=\left(\sigma_{2}-\sigma_{1}\right) \Delta(t, T),
\end{gathered}
$$

где

$$
\Delta(t, T)=-\Pi(t)+2 \Pi(t-T)-\Pi(t-2 T), t>2 T .
$$

Очевидно, что $\Delta(t, T) \geq 0$ для любого $t \geq 2 T$, так как $2 f(t-T)>f(t)+f(t-2 T)$ для любой выпуклой вверх функции; значит, $\varepsilon_{12}(t) \geq \varepsilon(t, \bar{\sigma}) \geq \varepsilon_{21}(t)$. Равенство имеет место только для вязкого элемента и модели Максвелла, для которых $\Delta(t, T) \equiv 0$ при $t>T$.

Докажем, что предел $\Delta(t, T)$ при $t \rightarrow \infty$ равен нулю. По теореме Лагранжа

$$
\Delta(t, T)=-\dot{\Pi}\left(t-\xi_{1}\right) T+\dot{\Pi}\left(t-\xi_{2}\right) T=\left(\dot{\Pi}\left(t-\xi_{2}\right)-\dot{\Pi}\left(t-\xi_{1}\right)\right) T,
$$

где $\xi_{1} \in(0, T), \xi_{1} \in(T, 2 T)$; так как существует предел $\dot{\Pi}(\infty)=v$, то $\Delta(t, T) \rightarrow v-$ $-v=0$ при $t \rightarrow \infty$. Из (13) следует, что при $t \rightarrow \infty$ всегда (для любой ФП и любого $v$ ) имеет место сходимость $\varepsilon_{12}(t, \bar{\sigma})-\varepsilon(t, \bar{\sigma}) \rightarrow 0, \quad \varepsilon_{21}(t, \bar{\sigma})-\varepsilon(t, \bar{\sigma}) \rightarrow 0$, где $\bar{\sigma}=$ $=\left(\sigma_{1}+\sigma_{2}\right) / 2$. Для произвольного $\sigma_{3} \neq \bar{\sigma}$ в случае $v \neq 0$ сходимости $\varepsilon_{12}\left(t, \sigma_{3}\right)-$ $-\varepsilon\left(t, \sigma_{3}\right) \rightarrow 0$ и $\varepsilon_{21}\left(t, \sigma_{3}\right)-\varepsilon\left(t, \sigma_{3}\right) \rightarrow 0$ не будет (см. п. 3$)$.

На рис. 3 приведены КП $\varepsilon_{12}(t, \bar{\sigma})$ и $\varepsilon_{21}(t, \bar{\sigma})$ для РеМ-4 с $\alpha=0,01, \beta=2$, $\gamma=1,5, \lambda=0,1$ для $T=10, \sigma_{1}=0,5, \sigma_{2}=1$ (черные КП), для модели Фойгта с $\beta=\gamma=$ $=1,5, \lambda=0,1, \alpha=0$ (красные КП), модели Максвелла с $\alpha=0,01, \beta=0,2$ (синие КП) и вязкого элемента с $\alpha=0,01, \beta=0$ (розовые КП). Штриховые линии - обычные КП $\varepsilon(t, \bar{\sigma})$. 


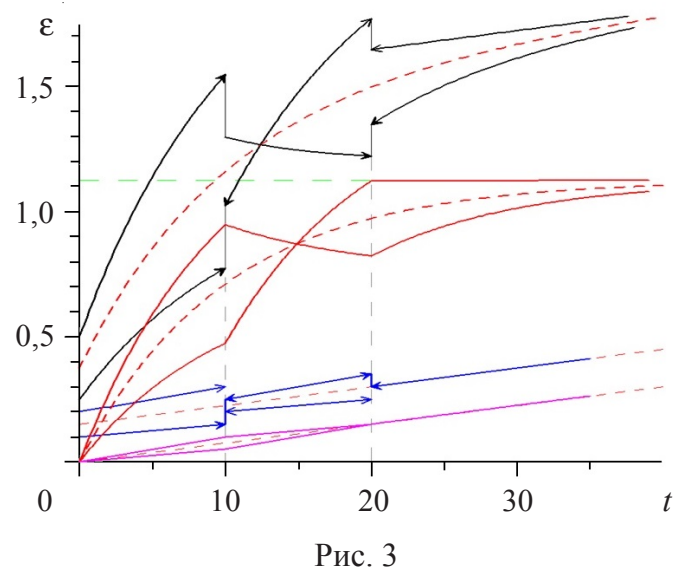

\section{6. Об условияХ затухания памяти в линейной вязкоупругости}

Утверждение о том, что убывания $\dot{R}(t)$ (или $\dot{\Pi}(t))$ «достаточно для выполнения условия затухания памяти» [14], нуждается в уточнении и корректировке. Кристенсен, ссылаясь на работы Вольтерры, Коулмэна и Нолла, формулирует условие затухания памяти (ЗП) весьма приблизительно: «Гипотеза о затухающей памяти утверждает, что отклик сильнее зависит от недавней истории изменения входного процесса, нежели от далекой истории..., то есть определяется некоторой весовой функцией, которая должна обеспечивать непрерывно убывающую зависимость от прошлых событий по мере их удаления от рассматриваемого момента времени». В ОС (4) эта «весовая функция» имеет вид $\dot{R}(t-\tau)$ (или $\dot{\Pi}(t-\tau))$, значит, $\dot{R}(t)$ и $\dot{\Pi}(t)$ должны убывать при $t>0$. Но утверждение [14], что этого «достаточно», неверно: убывание $\dot{R}(t)$ и П $(t)$ необходимо, но не достаточно, чтобы обеспечить даже интуитивное представление о ЗП, предполагающее, что материал по прошествии достаточно большого времени забудет о любом воздействии (возмущении) конечной длительности. Например, ФР модели Максвелла и РеМ-4 убывают очень быстро (экспоненциально), но, как показано выше, они бесконечно долго сохраняют накопленную к моменту разгрузки вязкую деформацию. Остаточная деформация накапливается у всех моделей с $v=\dot{\Pi}(\infty)>0$ (см. пп. 2,3): у них след, оставленный финитным импульсом нагрузки, не стирается никогда.

Убывание $\dot{R}(t)$ и $\dot{\Pi}(t)$ не стоит отождествлять с условием ЗП - тем более, что убывание $\dot{R}(t)$ наблюдается в испытаниях всех материалов. То свойство, о котором говорится в [14], можно назвать лишь монотонностью (или ослаблением) памяти, и оно является гораздо более всеобщим, чем свойства ЗП, выделяющие классы материалов (и операторы моделей) или только классы процессов, в которых ЗП проявляется. Один из разумных подходов к формализации свойства ЗП линейного оператоpa $\mathbf{A}$ в некотором счетно-нормированном пространстве функций на луче $[0, \infty)($ в частности, интегральных операторов $\Pi$ или $\mathbf{R}$ ОС (1)) намечен в $[6,8]$.

Определение. Оператор А обладает свойством затухающей памяти, если для $\forall T>0$ и любой пары (непрерывных) входных процессов, таких, что $x_{1}(t) \equiv x_{2}(t)$ при всех $t \geq T$ (для оператора на пространстве дифференцируемых функций можно еще потребовать дополнительно, чтобы $\left|\dot{x}_{1}(t)-\dot{x}_{2}(t)\right|<C$ при всех $\left.t \leq T\right)$, для их образов $y_{k}=\mathbf{A} x_{k}$ имеет место $y_{1}(t)-y_{2}(t) \rightarrow 0$ при $t \rightarrow \infty$. В силу линейности оператора $\mathbf{A}$ это равносильно тому, что для всякого процесса $x(t)$ с компактным 
носителем (заключенным в некотором отрезке $[0, T]$ ) его образ $y=\mathbf{A} x$ обладал свойством $y(t) \rightarrow 0$ при $t \rightarrow \infty$.

В этом определении зафиксировано самое слабое требование 3П, налагающее ограничение только на асимптотику отклика $y(t)[6]$.

Анализ свойств кривых релаксации с произвольной начальной стадией деформирования показал [23], что затухание памяти при релаксации имеет место всегда, то есть не требует дополнительных ограничений на функцию релаксации. Как установлено выше, условие $v=0$ необходимо для ЗП при ступенчатом нагружении. В работе [6] исследованы свойства кривых ползучести с произвольной начальной стадией нагружения $(\mathrm{HCH})$ и условия независимости их асимптотики от $\mathrm{HCH}$ (ее длительности и программы нагружения); в частности, доказано, что условие $v=0$ обеспечивает затухание влияния НСН с течением времени (стремление к нулю отклонения от КП при мгновенном нагружении), то есть является критерием затухания памяти при ползучести.

\section{Заключение}

Изучены общие математические свойства и качественные особенности семейств КП при любом ступенчатом нагружении, порождаемых ОС (1) с произвольной функцией ползучести, их зависимость от характеристик ФП и программ нагружения. Исследованы интервалы монотонности, скачки в точках разрыва и асимптотика КП, условия затухания памяти, влияние перестановки ступеней нагружения и т.п. В частности, установлено свойство асимптотической коммутативности КП, обнаружена ключевая роль величины предела производной ФП на бесконечности, доказано, что ее отличие от нуля влечет накопление остаточной деформации и отсутствие затухания памяти. Свойства теоретических КП сопоставлены с типичными свойствами экспериментальных КП вязкоупругопластичных материалов, выявлены эффекты, которые (не) способно описывать линейное ОС (1), и индикаторы его применимости.

\section{Список литературы}

1. Хохлов А.В. Качественный анализ линейных определяющих соотношений вязкоупругости. Свойства теоретических кривых деформирования, функиий ползучести и релаксации и их произведения: Отчет о НИР № 5218. НИИ механики МГУ им. М.В. Ломоносова, М. 2013. № ГР 01201152141. 93 с.

2. Хохлов А.В. Кривые обратной ползучести в рамках линейной вязкоупругости и необходимые ограничения на функцию ползучести. Проблемы прочности и пластичности. 2013. Вып. 75. Ч. 4. С. 257-267.

3. Хохлов А.В. Свойства произведения функции ползучести и функции релаксации в линейной вязкоупругости. Проблемы прочности и пластичности. 2014. Т. 76, №4. С. 343-356.

4. Хохлов А.В. Общие свойства диаграмм деформирования линейных моделей вязкоупругости при постоянной скорости деформации. Проблемы прочности и пластичности. 2015. Т. 77, №1. С. 60-74.

5. Хохлов А.В. Характерные особенности семейств кривых деформирования линейных моделей вязкоупругости. Проблемы прочности и пластичности. 2015. Вып. 77, № 2. С. $139-154$.

6. Хохлов А.В. Общие свойства семейств кривых ползучести при ступенчатом нагружении линейного определяющего соотношения вязкоупругости, условия моделирования эффекта Кольрауша и затухания памяти: Отчет о НИР №5254. НИИ механики МГУ им. М.В. Ломоносова, М. 2014. № ГР 01201152141. 83 c. 
7. Хохлов А.В. Качественный анализ определяющих соотношений как базовая стадия их аттестации. Современные проблемы математики, механики, информатики: Матер. Междунар. науч. конф. Тула: Изд-во ТулГУ, 2014. С. 435-438.

8. Хохлов А.В. Определяющее соотношение для реологических процессов: свойства теоретических кривых ползучести и моделирование затухания памяти. Изв. РАН. Механика твердого тела. 2007. №2. С. 147-166.

9. Хохлов А.В. Определяющее соотношение для реологических процессов с известной историей нагружения. Кривые ползучести и длительной прочности. Изв. РАН. Механика твердого тела. 2008. №2. С. 140-160.

10. Одквист Ф. Технические теории ползучести. Механика: Сб. переводов. 1959. №2. C. $101-111$.

11. Работнов Ю.Н. Ползучесть элементов конструкиий. М.: Наука, 1966. 752 с.

12. Бленд Д.Р. Теория линейной вязкоупругости. М.: Мир, 1965. 200 с.

13. Ильюшин А.А., Победря Б.Е. Основы математической теории термовязкоупругосmu. М.: Наука, 1970. 280 с.

14. Кристенсен Р. Введение в теорию вязкоупругости. М.: Мир, 1974. 338 с.

15. Работнов Ю.Н. Элементы наследственной механики твердых тел. М.: Наука, 1977. $384 \mathrm{c}$.

16. Малинин Н.Н. Расчеты на ползучесть элементов машиностроительных конструкций. М.: Машиностроение, 1981. 221 с.

17. Tschoegl N.W. The phenomenological theory of linear viscoelastic behavior. Berlin: Springer, 1989. $769 \mathrm{p}$.

18. Knauss W.G., Emri I., Lu H. Mechanics of polymers: viscoelasticity. Springer Handbook of Experimental Solid Mechanics. Ed. by W.N. Sharpe. N.Y.: Springer, 2008. P. 49-96.

19. Lakes R.S. Viscoelastic materials. UK: Cambridge Univ. Press, 2009. 462 p.

20. Георгиевский Д.В., Климов Д.М., Победря Б.Е. Особенности поведения вязкоупругих моделей. Изв. РАН. Механика твердого тела. 2004. №1. С. 119-157.

21. Dandrea J., Lakes R.S. Creep and creep recovery of cast aluminum alloys. Mechanics of Time-Dependent Materials. 2009. V. 13. P. 303-315.

22. Hamouda B.H., Laiarinandrasana L., Piques R. Viscoplastic behavior of a medium density polyethylene (MDPE): constitutive equations based on double nonlinear deformation model. Int. J. Plasticity. 2007. V. 23, No 8. P. 1307-1327.

23. Хохлов А.В. Свойства кривых релаксации с начальной стадией деформирования и затухание памяти в линейной теории вязкоупругости. Современные проблемы математики, механики, информатики: Матер. Междунар. науч. конф. Тула: Изд-во ТулГУ, 2014. C. 443-445.

\section{References}

1. Khokhlov A.V. Kachestvennyy analiz lineynykh opredelyayushchikh sootnosheniy vyazkouprugosti. Svoystva teoreticheskikh krivykh deformirovaniya, funktsiy polzuchesti i relaksatsii i ikh proizvedeniya: Otchet o NIR № 5218. NII mekhaniki MGU im. M.V. Lomonosova, M. 2013. № GR 01201152141.93 s.

2. Khokhlov A.V. Krivye obratnoy polzuchesti v ramkakh lineynoy vyazkouprugosti i neobkhodimye ogranicheniya na funktsiyu polzuchesti. Problemy prochnosti i plastichnosti. 2013. Vyp. 75. Ch. 4. S. 257-267.

3. Khokhlov A.V. Svoystva proizvedeniya funktsii polzuchesti i funktsii relaksatsii v lineynoy vyazkouprugosti. Problemy prochnosti i plastichnosti. 2014. T. 76, №4. S. 343-356.

4. Khokhlov A.V. Obshchie svoystva diagramm deformirovaniya lineynykh modeley vyazkouprugosti pri postoyannoy skorosti deformatsii. Problemy prochnosti i plastichnosti. 2015. T. 77, №1. S. 60-74.

5. Khokhlov A.V. Kharakternye osobennosti semeystv krivykh deformirovaniya lineynykh modeley vyazkouprugosti. Problemy prochnosti i plastichnosti. 2015. Vyp. 77, № 2. S. 139-154.

6. Khokhlov A.V. Obshchie svoystva semeystv krivykh polzuchesti pri stupenchatom nagruzhenii lineynogo opredelyayushchego sootnosheniya vyazkouprugosti, usloviya modelirovaniya 
effekta Kol'rausha i zatukhaniya pamyati: Otchet o NIR №5254. NII mekhaniki MGU im. M.V. Lomonosova, M. 2014. № GR 01201152141. 83 s.

7. Khokhlov A.V. Kachestvennyy analiz opredelyayushchikh sootnosheniy kak bazovaya stadiya $\mathrm{ikh}$ attestatsii. Sovremennye problemy matematiki, mekhaniki, informatiki: Mater. Mezhdunar. nauch. konf. Tula: Izd-vo TulGU, 2014. S. 435-438.

8. Khokhlov A.V. Opredelyayushchee sootnoshenie dlya reologicheskikh protsessov: svoystva teoreticheskikh krivykh polzuchesti i modelirovanie zatukhaniya pamyati. Izv. RAN. Mekhanika tverdogo tela. 2007. №2. S. 147-166.

9. Khokhlov A.V. Opredelyayushchee sootnoshenie dlya reologicheskikh protsessov c izvestnoy istoriey nagruzheniya. Krivye polzuchesti i dlitel'noy prochnosti. Izv. RAN. Mekhanika tverdogo tela. 2008. №2. S. 140-160.

10. Odkvist F. Tekhnicheskie teorii polzuchesti. Mekhanika: Sb. perevodov. 1959. №2. S. $101-111$.

11. Rabotnov Yu.N. Polzuchest' elementov konstruktsiy. M.: Nauka, 1966. 752 s.

12. Blend D.R. Teoriya lineynoy vyazkouprugosti. M.: Mir, 1965. $200 \mathrm{~s}$.

13. Il'yushin A.A., Pobedrya B.E. Osnovy matematicheskoy teorii termovyazkouprugosti. M.: Nauka, 1970. $280 \mathrm{~s}$.

14. Kristensen R. Vvedenie v teoriyu vyazkouprugosti. M.: Mir, 1974. 338 s.

15. Rabotnov Yu.N. Elementy nasledstvennoy mekhaniki tverdykh tel. M.: Nauka,1977. $384 \mathrm{~s}$.

16. Malinin N.N. Raschety na polzuchest' elementov mashinostroitel'nykh konstruktsiy. M. Mashinostroenie, 1981.221 s.

17. Tschoegl N.W. The phenomenological theory of linear viscoelastic behavior. Berlin: Springer, 1989. $769 \mathrm{p}$.

18. Knauss W.G., Emri I., Lu H. Mechanics of polymers: viscoelasticity. Springer Handbook of Experimental Solid Mechanics. Ed. by W.N. Sharpe. N.Y.: Springer, 2008. P. 49-96.

19. Lakes R.S. Viscoelastic materials. UK: Cambridge Univ. Press, 2009. 462 p.

20. Georgievskiy D.V., Klimov D.M., Pobedrya B.E. Osobennosti povedeniya vyazkouprugikh modeley. Izv. RAN. Mekhanika tverdogo tela. 2004. №1. S. 119-157.

21. Dandrea J., Lakes R.S. Creep and creep recovery of cast aluminum alloys. Mechanics of Time-Dependent Materials. 2009. V. 13. P. 303-315.

22. Hamouda B.H., Laiarinandrasana L., Piques R. Viscoplastic behavior of a medium density polyethylene (MDPE): constitutive equations based on double nonlinear deformation model. Int J. Plasticity. 2007. V. 23, No 8. P. 1307-1327.

23. Khokhlov A.V. Svoystva krivykh relaksatsii s nachal'noy stadiey deformirovaniya i zatukhanie pamyati v lineynoy teorii vyazkouprugosti. Sovremennye problemy matematiki, mekhaniki, informatiki: Mater. Mezhdunar. nauch. konf. Tula: Izd-vo TulGU, 2014. S. 443-445.

\title{
PROPERTIES OF CREEP CURVES AT PIECEWISE-CONSTANT STRESS GENERATED BY THE LINEAR VISCOELASTICITY THEORY
}

\author{
Khokhlov A.V. \\ Institute of Mechanics, Lomonosov Moscow State University, \\ Moscow, Russian Federation
}

The qualitative analysis of the linear integral constitutive relation of viscoelasticity with an arbitrary (increasing convex-up) creep compliance function is continued. General equation and basic properties of theoretic creep curves at piecewise-constant stress generated by linear constitutive equation are analytically studied herein, their dependence on creep compliance function and loading program parameters is analyzed. Relations between stress and strain jumps, extremum points of creep curves and their asymptotic behavior at infinity, conditions of fading memory, influence of stress steps permutation, asymptotic commutativity are considered. The governing role of a creep function derivative limit value at infinity is revealed. It is proved for any increasing convex-up creep function that non-zero value of its derivative limit implies accumulation of plastic (non- 
recoverable) strain and lack of fading memory property. The main classes of linear models (i.e. regular, unbounded and singular) are considered and distinctive features of their creep curves are marked.

General qualitative properties of theoretic creep curves are compared to typical test creep curves of viscoelastoplastic materials in order to examine linear theory abilities to provide an adequate description of basic rheological phenomena related to creep, recovery and cyclic step-wise loading. The specific features of creep curves produced by the linear integral constitutive equation are highlighted that can be used as indicators marking the field of its applicability or non-applicability in examination of a certain material test curves.

Keywords: creep compliance, integral constitutive equation, theoretic creep curves at piecewiseconstant loading, creep curves asymptotics, fading memory, asymptotic commutativity, regular and singular models, adequacy indicators for linear viscoelasticity. 